Pacific Journal of Mathematics

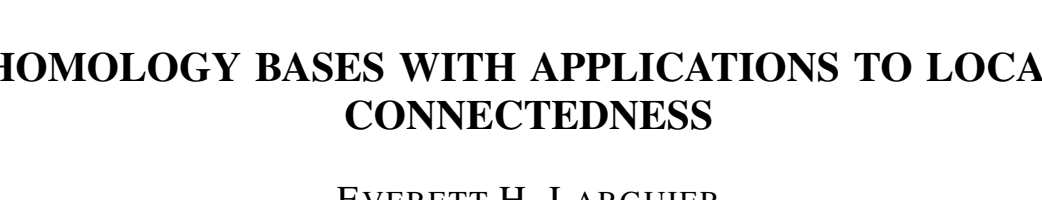




\title{
HOMOLOGY BASES WITH APPLICATIONS TO LOCAL CONNECTEDNESS
}

\author{
EVERETT LARGUIER
}

\section{INTRODUCTION}

1. The present investigation originated in an attempt to extend certain results of Wilder [11] concerning the imbedding of compact metric spaces in locally connected spaces. The means that suggested themselves for the treatment of the problem necessitated an analys is of the homology group of a compact space with respect to certain sequences of direct-sum decompositions. In the earlier part of this paper we introduce the concept of sequential decomposability of a vector space with respect to a countable set of subspaces. An equivalence between sequential decompositions is set up with the usual properties. Decomposition theorems with uniqueness properties for elements of the vector space are noted. In terms of a nonnegative real-valued function on elements of the vector space, a topology is introduced in which the vector space is a Hausdorff space if and only if it is metric; the completeness of this space is also discussed. When the vector space is a complete metric space, there exists a countable set of elements associated with the sequential decomposition which becomes a base for the space in the sense of infinite combinations.

Since the homology group of a compact space with coefficients over a field is a vector space, the preceding results are applicable; conditions are discussed under which the homology group is a metric space, and further conditions under which it is complete. The existence problem arising here is disposed of readily by showing that compact $G_{\delta}$ subsets of locally compact $\mathrm{lc}^{r}$ spaces (see 8.1 below) satisfy the requirements of the preceding abstract situation. This gives further an intrinsic approach to the existence of a fundamental system of 0 cycles discussed by Wilder in another setting [13, Ch. VI, \$5].

In an investigation of this kind it is often desirable from many points of view to have a specialized class of objects with a well-defined property; hence we next occupy ourselves with determining the existence of a fundamental

Received August 7, 1951. Presented to the American Mathematical Society, September 4, 1947. The author acknowledges with gratitude the guidance and direction of Professor R.L. Wilder during the course of this investigation; to Professor Steenrod also thanks are due for many profitable conferences.

Pacific J. Math. 2 (1952), 191 - 208 
system of nontrivial 0 -cycles for the sets described above. The existence of such a fundamental system plays a mildly important role in the sequel.

With this done, the stage is set for a partial solution of the problem whence arose the whole discussion, and a sufficient condition is given under which a compact Hausdorff space $M$ is imbeddable as a $G_{\delta}$ subset of a $0-1$ c continuum, which is obtained by adjoining a denumberable infinitude of disjoint arcs to M. To improve the value of the preceding result, it is pointed out that the hypothesis of the theorem is verified by a class $\hat{\ell}$ of compact spaces which includes definitely nonmetric spaces. Using the same technique as was employed in obtaining the preceding result, we show further that the class $\Re$ contains as a proper subset the class of all compacta. Another condition is given that a separable compact $G_{\delta}$ subset of a space $S$ be a subset of a locally compact subset $S^{\prime} \subset S$. A localization of the preceding condition gives the last application of the alsstract development.

\section{Some Propertifs of Vector Spaces}

2. Sequential decomposition. In the sequel all vector spaces will be taken over a given field, $\tau$. Let $\mathbf{H}=\left\{H_{k}\right\}$ be a monotonic decreasing sequence of subspaces of a vector space $V$ such that each $I_{k}$ is finite co-dimensional [4, Ch. 2, Def. 2], then evidently [8, Ch. II, (23.1)] there always exists a countable set of linearly independent elements $\mathbf{g}=\left\{g_{i}\right\}$ of $V$ such that $(\mathrm{i}) \mathbf{g}_{k}=\left\{g_{i} \mid 1 \leq\right.$ $\left.i \leq i_{k}\right\}$ is a set of generators for a subspace $G_{k}$, (ii) $V$ is the direct sum of $G_{k}$ and $H_{k}$, and (iii) $\left\{g_{i} \mid i>i_{k}\right\} \subset H_{k}$. Similarly, if $\mathbf{g}=\left\{g_{i}\right\}$ is a countable linearly independent set of elements of a vector space $V$ then there exists a sequence $\mathbf{H}$ of subspaces having the properties described above such that (i), (ii), and (iii) again hold. This pair $(\mathbf{H}, \mathbf{g})$ will be called a sequential decomposition of of $V$. Evidently $\mathbf{g}$ is not unique.

In terms of a fixed sequential decomposition we may obviously [8, Ch. II, (23.1)] assert that to each element $v \in V$ there corresponds a unique infinite linear form which we may denote by

$$
\sum_{i=1}^{\infty} a^{i} g_{i} \text {, where } v=\sum_{i=1}^{i_{k}} a^{i} g_{i}\left(\bmod H_{k}\right)
$$

for each positive integer $k, a^{i} \in \mathfrak{F}$. The $\sum_{i=1}^{i_{k}} a^{i} g_{i}$ will be called the $k$-decomposition ( $\operatorname{rel} \mathbf{H}, \mathbf{g})$ of $v \in V$. We note that at this stage of the development it may, and at times certainly will, happen that the same linear form will correspond to distinct elements of $V$. Evidently if to two elements $v_{1}, v_{2} \in V$, there corresponds the same infinite linear form, then $v_{1}=v_{2}\left(\bmod H_{k}\right)$ for each $k$. 
3. 'ropology for the vector space. W'e now define the positive real-valued function $\rho$ on the product space $V \times V$, relative to a fixed sequential decomposition $(\mathbf{H}, \mathbf{g})$, as follows: if $v_{1}, v_{2} \in V$, then (1) $\rho\left(v_{1}, v_{2}\right)=1 / k$ for some positive integer $k \Longleftrightarrow k$ is the largest index such that $v_{1}=v_{2}\left(\bmod H_{k}\right)$; (2) if there is no upper bound to the $k$ 's such that $v_{1}=v_{2}\left(\bmod H_{k}\right)$, then $\rho\left(v_{1}, v_{2}\right)=0$. It is quite simple to prove that:

$$
\rho\left(v_{1}, v_{2}\right)=\rho\left(v_{2}, v_{1}\right)
$$

and

$$
\rho\left(v_{1}, v_{3}\right) \leq \rho\left(v_{1}, v_{2}\right)+\rho\left(v_{2}, v_{3}\right)
$$

We note, in view of an earlier comment, that $\rho\left(v_{1}, v_{2}\right)=0$ does not necessarily imply $v_{1}=v_{2}$. Hence it is clear that the function $\rho$ cannot always serve as a metric for the vector space $V$. Under suitable conditions this will be possible, however, as will be noted below. At this stage $\rho$ may be called a pseudo-metric.

For each $v \in V$ and each positive integer $k$, the family of sets of the form

$$
U(v)=\left\{v^{\prime} \mid \rho\left(v^{\prime}, v\right)<i / k\right\}
$$

will, by finite intersection and arbitrary union, induce a topology for $V$ in which the family above is a family of open sets. With this topology, $V$ is not necessarily a Hausdorff space [see, for example 8, p.25]; however, it is readily seen that in order that $V$ be Hausdorff it is necessary and sufficient that it be metrizable by the function $\rho$. Then we may write

$$
v=\sum_{i=1}^{\infty} a^{i} g_{i}=\lim _{k \rightarrow \infty} \sum_{i=1}^{i_{k}} a^{i} g_{i}, \quad a^{i} \in \tilde{\xi}
$$

We shall call $(\mathbf{H}, \mathbf{g})$ and $\left(\mathbf{H}^{\prime}, \mathbf{g}^{\prime}\right)$ equivalent sequential decompositions if, when $\mathbf{H}=\left\{H_{k}\right\}$ and $\mathbf{H}^{\prime}=\left\{H_{j}^{\prime}\right\}$, for each $k$ there exists a $j$ such that $H_{k} \supset H_{j}^{\prime}$, and conversely. It is relatively easy to show that if $(\mathbf{H}, \mathbf{g})$ and $\left(\mathbf{H}^{\prime}, \mathbf{g}^{\prime}\right)$ are equivalent sequential decompositions and $J$ and ${ }^{\prime} J^{\prime}$ and $\rho$ and $\rho^{\prime}$ are respectively topologies and pseudo-metrics relative to $(\mathbf{H}, \mathbf{g})$ and $\left(\mathbf{H}^{\prime}, \mathbf{g}^{\prime}\right)$, then, when $V$ is Hausdorff in both cases, the topologies and the metrics are equivalent in the usual sense. The proof in this instance proceeds along lines usual in such cases.

4. A fundamental system for $V$. When $V$ is a Hausdorff space in the topology described above, then we may assert the following readily proved properties: 
(1) For any nonzero element a $\in \mathcal{F}$, and any $v \in V$, we have $\rho(a v, 0)=\rho(v, 0)$.

(2) If $(\mathbf{H}, \mathbf{g})$ is the sequential decomposition, then $\lim _{i \rightarrow \infty} g_{i}=0$.

(3) If $(\mathbf{H}, \mathbf{g})$ is the sequential decomposition, and

$$
v_{1}=\sum_{i=1}^{\infty} a^{i} g_{i} \text { and } v_{2}=\sum_{i=1}^{\infty} \mathfrak{b}^{i} g_{i} \text {, }
$$

then $v_{1} \neq v_{2} \Longleftrightarrow a^{i} \neq \mathfrak{G}^{i}$ for some $i$.

(4) If furthermore $V$ is the complete enclosure [10, p. 28] of the vector space $G$ spanned by $\mathbf{g}$, then $G$ is dense in $V$ and, in addition to the preceding, to each infinite linear form $\sum a^{i} g_{i}, a^{i} \in \mathcal{F}$ and $g_{i} \in \mathbf{g}$, there corresponds a unique element of $V$.

When this last property is satisfied, the set $\mathbf{g}$ becomes a sort of base for the space $V$, and it will be called a fundamental system for $V$. With appropriate particularization, this is identical with the fundamental system of $r$-cycles treated by Wilder [13, Ch. VI, §5]. Later results of this paper will give a new intrinsic approach to the problem of the existence of this system of cycles.

\section{HomOLOGY BASES}

5. Preliminaries. The homology theory used in this paper is the usual Čech homology theory, originally formulated in [6], augmented in the sense of [8, p. 248]. We recall, for later clarity, that if $U$ is a covering of a space, then the nerve of the covering $U$, written $N(U)$, is the abstract simplicial complex defined as follows: Every element of the covering is a vertex of the nerve, and for each natural number $k$ every set of $k+1$ elements with a nonvacuous intersection forms a $k$-simplex of the nerve. We shall consistently use lower case Greek or Roman letters to denote individual cycles: thus $z^{r}$ is an $r$-cycle; capitals will indicate homology classes of cycles, thus $Z^{r} ; z^{r}(U)$ will denote the representative on the nerve of the covering $U$ of the cycle $z^{r}$; likewise if $H_{k}^{r}$ is a subset of $H^{r}(S, \mathcal{F})$, then $H_{k}^{r}(U)$ is the corresponding subset of the homology group $H^{r}(U)$ on the nerve of the covering $U$; and so on. Finally, $\pi_{u v}$ will be the usual projection mapping. Note that here, as well as later, subscripts referring to a covering, say $U$, will be printed as the corresponding Roman letter; this is done for obvious typographical reasons.

If $\mathcal{S}^{r}=\left\{H^{r}(U)\right\}$ is the inverse system of homology groups, with coefficients from a field $\mathfrak{F}$, on nerve complexes for the family of all finite coverings of a compact space $S$, then the limit group is the homology group $H^{r}(S, \mathfrak{F})$ of the 
space $S$. The limit group, and also the homology groups of the nerve complexes, are vector spaces; hence all that has been noted in the preceding section is applicable here.

In terms of the notation found in [13, p. 147], $h_{u v}$ will be the homomorphism of $H^{r}(Y)$ in $I^{r}(U)$ induced by the projection of $N\left(U^{Y}\right)$ in $N(U)$. Te shall further use $h_{u}$ to denote the homomorphism which associates with an element of the homology group of the space its representative in $I I^{r}(U)$. Evidently, if $b^{*}>\langle i$ then $h_{u w}=h_{u v} h_{v w}$ and $h_{u}=h_{u v} h_{v}$.

5.1. UEFINITION. A sequential decomposition $\left(\mathbf{H}^{r}, \Gamma^{r}\right)$ is compatible with a sequence of coverings $\left\{\varepsilon_{k}\right\}$ if and only if $I_{k}$ is the kernel of $h_{e_{k}}$.

The existence of a sequential decomposition compatible with a given sequence of coverings is obvious. Concerning this notion we make the following remarks which will be used later.

5.2. REMARK. If $\left(\mathbf{H}^{r}, \Gamma^{r}\right)$ and $\left\{\varepsilon_{k}\right\}$ are compatible, then the sequence of representative cycles, $\gamma^{r}$ has the following properties: (i) the subset of $\gamma^{r}\left(\varepsilon_{k}\right)$ defined by $\left\{\gamma_{i}^{r}\left(\varepsilon_{k}\right) \mid 1 \leq i \leq i_{k}\right\}$ is lirh (that is, linearly independent relative to homology) on $N\left(\varepsilon_{k}\right)$ for each $k$; and (ii) $\gamma_{i}^{r}\left(\varepsilon_{k}\right) \sim 0$ on $N\left(\varepsilon_{k}\right)$ for $i>i_{k}$.

5.3. REMARK. If $\left(\mathbf{H}^{r}, \Gamma^{r}\right)$ and $\left\{\varepsilon_{k}\right\}$ are compatible, and $Z_{1}^{r}, Z_{2}^{r} \in I^{r}(S, \tau)$, then $\rho\left(Z_{1}^{r}, Z_{2}^{r}\right)=1 / k \Longleftrightarrow k$ is the largest index such that $z_{1}^{r}-z_{2}^{r}$ belongs to the kernel of the homomorphism $h_{e_{k}}$.

6. Metrizability. In the preceding, part on vector spaces it was noted that a necessary and sufficient condition for the vector space to be llausdorff under the topology introduced was the metrizability by the function $\rho$. We shall formulate here a sufficient condition that $I^{r}(S, \mathcal{F})$ be metrizable in this way. As a preliminary we introduce the following lemma.

6.1. LEMMA. (i) Let there exist for each covering $U$ of $S$ a number $k(U)$ and a refinement $U^{\prime}>U$ such that $H_{k(u)}^{r}\left(U^{\prime}\right)$ belongs to the kernel of $n_{u u^{\prime}}$; and (ii) let $\Gamma^{r} \in H^{r}(S, \xi)$ be such that for each $k$ there exists a covering $V(k)$ with the property that

$$
V^{\prime}>V(k) \Rightarrow h_{v}^{\prime} \Gamma^{r} \subset h_{v^{\prime}} H_{k}^{r}=H_{k}^{r}\left(U^{\prime}\right)
$$

Then $\Gamma^{r}=0$.

Proof. Let $U$ be an arbitrary covering of $S$; we must show that $h_{u} \Gamma^{r}=0$. Let $k(U)$ be the number given by hypothesis (i), and $U^{\prime}$ the refinement such that $h_{u}{ }^{\prime} H_{k(u)}^{r}$ belongs to the kernel of $h_{u u^{\prime}}$. By hypothesis (ii), for $k=k(U)$, 
we have $U(k)$ such that

$$
U^{\prime}>V(k) \Longrightarrow h_{v}, \Gamma^{r} \subset h_{v}, H_{k}^{r}
$$

Let $U$ be a common refinement of $U^{\prime}$ and $V(k)$. Then we have

$$
h_{u w} h_{w} H_{k}^{r}=h_{u u^{\prime}} h_{u^{\prime}}{ }^{\prime} h_{w} H_{k}^{r}=h_{u u^{\prime}} h_{u}, H_{k}^{r}=0
$$

But $h_{w} \Gamma^{r} \subset h_{w} H_{k}^{r}$. lience we evidently have the desired result.

Wie may now state and prove a theorem giving a sufficient condition for the metrizability of $H^{r}(S, \mathfrak{F})$.

6.2. THEOREM. If for each covering $U$ of $S$ the hypothesis (i) of the preceding lemma is satisfied, then $H^{r}(S, \mathcal{F})$ is metrizable.

Proof. Evidently all we need prove is that, for $Z_{1}^{r}, Z_{2}^{r} \in H^{r}(S, \mathcal{\mho})$, we have $\rho\left(Z_{1}^{r}, Z_{2}^{r}\right)=0 \Longrightarrow Z_{1}^{r}=Z_{2}^{r}$. Now $\rho\left(Z_{1}^{r}, Z_{2}^{r}\right)=0$ implies that $Z_{1}^{r}-Z_{2}^{r} \in H_{k}^{r}$ for each $k$. But then [13, p. 147] for each covering $U$ of $S$ we have $h_{u}\left(Z_{1}^{r}-Z_{2}^{r}\right) \subset$ $h_{u} H_{k}^{r}$, and thus hypothesis (ii) of the preceding lemma is more than satisfied, so that $Z_{1}^{r}-Z_{2}^{r}=0$ as required.

7. Completeness. Before establishing the completeness of $H^{r}(S, \mathfrak{F})$, we introduce the following definition for convenience of reference.

7.1. DEFINITION. A compact space $S$ will be said to have property $P_{r}$ (rel $\left.\mathbf{H}^{r}, \Gamma^{r}\right)$ if and only if for each covering $U$ of $S$ there exists an index $k=k(U)$ such that $H_{k}^{r}$ is contained in the kernel of $h_{u}$. A space $S$ will be said to have the property $P_{r}\left(\operatorname{rel} \mathbf{H}^{r}, \Gamma^{r}\right)$ with respect to a compact subset $M \subset S$, if the property described is satisfied with respect to the homology theory of $M$ itself.

The following lemmas are quite obvious.

7.2. LEMMA. If the compact space $S$ has property $P_{r}\left(\operatorname{rel} \mathbf{H}^{r}, \Gamma^{r}\right)$, then hypothesis (i) of Lemma 6.1 is satisfied.

7.3. LEMMA. If $U>U$ and $k^{\prime}(U)$ and $k^{\prime}(U)$ are respectively the least indices greater than 1 for which $H_{k}^{r}$ is contained in the kernel of $h_{v}$ and $h_{u}$, then $k^{\prime}(U) \geq k^{\prime}(U)$.

7.4. THEOREM. If the compact space $S$ has property $P_{r}\left(\operatorname{rel} \mathbf{H}^{r}, \Gamma^{r}\right)$, then $H^{r}(S, \mathcal{\mho})$ is a complete metric space.

Proof. By I,emma 7.2 and Theorem 6.2, metrizability follows obviously. Let 
$\left\{Z_{i}^{r}\right\}$ be a Cauchy sequence of elements of $H^{r}(S, \widetilde{F})$, and let $\mathbf{U}$ be a complete family of coverings of $S$. For each $U \in \mathbf{U}$ let $k(U)$ be the smallest index greater than 1 for which the defining condition of property $P_{r}$ holds. Let $j(U)$ be the smallest natural number such that for all $s$ we have $Z_{j(u)}^{r}-Z_{j(u)+s}^{r} \in H_{k(u)}^{r}$. We now let $z^{r}(U)=z_{j(u)}^{r}(U)$. We shall first show that the $z^{r}$ thus defined by its coordinates on a complete family of coverings is a cycle.

Suppose $U, U \in \mathbf{U}$ such that $V>U$; we must show that $\pi_{u v} z^{r}(U) \sim z^{r}(U)$. As a consequence of Lemma 7.3, we need only consider the single case $k(U) \geq$ $k(U)$. Under this hypothesis, we have $j(U) \geq j(U)$, and so we have $z_{j(u)}^{r}(U)-$ $z_{j(v)}^{r}(U) \sim \phi^{r}(U), \Phi^{r} \in H_{k}^{r}$; hence $z_{j(u)}^{r}(U) \sim z_{j(v)}^{r}(U)$. But $\pi_{u v} z_{j(v)}^{r}(U) \sim$ $z_{j(v)}^{r}(U)$. Therefore, as was required, $\pi_{u v} z_{j(v)}^{r}(U) \sim z_{j(u)}^{r}(U)$. Hence $z^{r}(U)$ is the coordinate on $N(U)$ of a cycle $z^{r}$ as defined above.

We now complete the proof by showing that $\lim _{i \rightarrow \infty} Z_{i}^{r}=Z^{r}$. Let $n_{k}$ be the smallest natural number such that $\rho\left(Z_{i}^{r}, Z_{j}^{r}\right) \leq 1 / k$ ( $k$ fixed) for all $i, j \geq$ $n_{k}$. Let $\mathbf{U}^{\prime}$ be a subset of $\mathbf{U}$ such that for $U \in \mathbf{U}^{\prime}$ the $k(U)$ as defined above in this proof is greater than or equal to $k$. Hence $j(U) \geq n_{k}$, and thus $Z_{j(u)}^{r}(U)-$ $Z_{i}^{r}(U) \in h_{u} H_{k}^{r}$ for all $i \geq h_{k}$. Now, in virtue of Lemma 7.3, it is evident that $\mathrm{U}^{\prime}$ is a complete family of coverings, so that the proof of the theorem is complete.

In this connection we might note the following rather obvious consequences of the preceding result.

7.5. When the compact space $S$ has the property $P_{r}\left(\mathrm{rel} \mathbf{H}^{r}, \Gamma^{r}\right)$, then

(1) for each $r$-cycle $z^{r}$ of $S$ there exists a unique homology relation of the form

$$
z^{r} \sim \sum_{i=1}^{\infty} a^{i} \gamma_{i}^{r},
$$

(2) $H^{r}(S, \mathfrak{\xi})$ is the complete enclosure of the vector space $H_{\Gamma}^{r}(S, \mathfrak{F})$ generated by $\Gamma^{r}$;

(3) $H_{\Gamma}^{r}(S, \mathfrak{f})$ is dense in $H^{r}(S, \mathfrak{F})$;

(4) $H^{r}(S, \tilde{\xi})$ is equivalent to the product space $P=P_{1}^{r} \times P_{2}^{r} \times \cdots \times P_{i}^{r} \times \cdots$, $P_{i}^{r}=\left\{\Gamma^{r} \mid \Gamma^{r}=a \Gamma_{i}^{r}, a \in \mathcal{F}\right\}$.

\section{Existence TheOREMS}

8. Locally connected spaces. We proceed now to establish the existence of a situation in which the preceding formal development is completely verified. 
The results of this section will, further, constitute a new intrinsic approach to the problem of the existence of fundamental systems of $r$-cycles treated in [13, Ch. VI, \$5].

8.1. Definition. A space $S$ is $r$-lc at a point $x \in S$, if and only if each open set which contains $x$ also contains a pair of open sets, $P, Q$, such that (i) $x \in Q \subset P$, and (ii) the number of $r$-cycles on $Q$ that are lirh on $P$ is finite, $A$ space $S$, is $r$-lc if and only if it is $r$-lc at each $x . S$; and finally $S$ is $\mathrm{lc}^{r}$, if and only if it is $p-\mathrm{lc}$ for each $p$ such that $0 \leq p \leq r$.

As an immediate consequence of the remarks made heretofore and a theorem proved elsewhere [13, p. 186, Th. 5.1], we have the following result.

8.2. ThEOREM. (i) Let $M$ be a compact $G_{\delta}$ subset of a locally compact $\mathrm{Ic}^{r}$ space $S$, and (ii) let $\left\{U_{k}\right\}$ be a sequence of open sets such that $U_{k+1} \mathbb{C} U_{k}$ and $\cap U_{k}=M$. Then there exists a sequential decomposition $\left(H^{r}, \Gamma^{r}\right)$ of $H^{r}(M, \mathfrak{F})$ such that: (1) $\Phi_{k}^{r} \in H_{k}^{r} \Longleftrightarrow \Phi_{k}^{r} \sim 0$ on $\bar{U}_{k}$; (2) $\left\{\gamma_{i}^{r} \mid 1 \leq i \leq i_{k}\right\}$ is a basis for cycles of $M$ with respect to homologies on $\bar{U}_{k}$.

The sequential decomposition given by the preceding theorem will be called compatible with the sequence of open sets $\left\{U_{k}\right\}$.

8.3. TheоREM. Let $M \subset S$ satisfy hypothesis (i), and let $\left\{U_{k}\right\}$ and $\left\{U_{j}^{\prime}\right\}$ be two sequences of open sets satisfying hypothesis (ii) of Theorem 8.2. Let $\left(\mathbf{H}^{r}, \Gamma^{r}\right)$ and $\left(\mathbf{H}^{\prime r}, \Gamma^{\prime r}\right)$ be compatible sequential decompositions given by the conclusion of Theorem 8.2. Then the sequential decompositions are equivalent.

Proof. For each $k$, there exists a $j$ such that $U_{k} \supset U_{j}$. By hypothesis, $\Phi_{j}^{\prime r} \in H_{j}^{\prime r} \Longrightarrow \Phi_{j}^{\prime r} \sim 0$ on $\bar{U}_{j}^{\prime r}$ and so also on $\bar{U}_{k}$; and $H_{k}^{r}$ is the subspace consisting of all classes of $r$-cycles which have representatives homologous to zero on $\bar{U}_{k}$; hence $\Phi_{j}^{\prime r} \in H_{k}^{r}$, so that $H_{j}^{\prime r} \subset H_{k}^{r}$ as required. The converse procedure completes the proof that the sequential decompositions are equivalent.

Because it will be used frequently in the sequel, we formulate the following hypothesis here for convenience of reference.

8.4. НY РотнеSIS. Let $M$ be a compact $G_{\delta}$ subset of a locally compact $\operatorname{lc}^{r}$ space $S$, and let $\left\{U_{k}\right\}$ be a sequence of open sets such that $U_{k} \supseteq U_{k+1}$ and $\cap U_{k}=M$; further let $\left(\mathbf{H}^{r}, \Gamma^{r}\right)$ be a sequential decomposition compatible with $\left\{U_{k}\right\}$.

We now treat the possibility of obtaining a sequence $\left\{\varepsilon_{k}\right\}$ of coverings of $S$ compatible with a given sequential decomposition. 
8.5. THEOREM. Let hypothesis 8.4 be satisfied. Then there exists a sequence $\left\{\varepsilon_{j}\right\}$ of coverings of $S$ compatible with a sequential decomposition $\left(\mathbf{H}^{\prime r}, \Gamma^{\prime r}\right)$ of $H^{r}(M, \widetilde{\xi})$ such that $\left(\mathbf{H}^{\prime r}, \Gamma^{\prime r}\right)$ and $\left(\mathbf{H}^{r}, \Gamma^{r}\right)$ are equivalent.

Proof. Let $\varepsilon_{1}$ be a covering of $S$ given by a well-known lemma [8, p. 263; or 13, p. 134, Lemma 8.7], due originally to $\check{C}^{2} h_{\text {, such that }} \mathscr{\varepsilon}_{1}$ is a refinement of a covering $U\left[13\right.$, p. 167, Theorem 19.2], such that $\gamma_{1}^{r}(U), \cdots, \gamma_{i_{1}}^{r}\left(U^{Y}\right)$ are lirh on $N(U)$ for all $U>U$. Let $P$ be the open set containing $M$ such that if the nucleus of a cell of $N\left(\varepsilon_{1}\right)$ is on $\bar{P}$, then it is on 1 . There exists a $k$ such that a subset $P^{*} \subset P$ has the additional property that $U_{k-1} \supseteq P^{*} \supseteq U_{k}$. Since $I H_{k}^{r}$ consists of classes of $r$-cycles of $M$ which are homologous to zero on $U_{k}$, it is evident that $h_{k}^{r}$ is contained in the kernel of $h_{e_{1}}$. Leet us designate the kernel of $h_{e_{1}}$ by $I_{1}^{\prime r}$. Since $\varepsilon_{1}>U$, we have the following inclusions: $H_{1}^{r}$ ว kernel of $h_{u} \supset H_{1}^{\prime r}$.

Evidently we may repeat this procedure and eventually obtain a sequence of coverings $\left\{\varepsilon_{j}\right\}$ of $S$ satisfying the following: (1) only a finite number of elements of $\varepsilon_{j}$ meet $M$ for each $j ;(2)$ for each $j$ there exists a $k_{j}$ such that $H_{j}^{\prime r} \supset H_{k_{j}}^{r}$; and (3) conversely, for each $k$ there is a $j_{k}$ such that $H_{k}^{r} \supset H_{j}^{\prime r}$. Consequently the sequential decompositions are equivalent as required.

9. Existence of a fundamental system. This section will be devoted to the proof of the existence of a fundamental system of $r$-cycles.

9.1. THEOREM. Let hypothesis 8.4 be verified. Then the space $S$ has the property $P_{r}$ (rel $\mathbf{H}^{r}, \Gamma^{r}$ ) with respect to the subset $M$.

Proof. There exists a complete family $\mathbf{U}$ of coverings of $S$ satisfying the following conditions: (1) if $U \in \mathbf{U}$, then at most a finite number of elements of $U$ meet $M$; (2) there exists an open set $P \supset M$ such that if the nucleus of a cell of $N(U)$ meets $P$, then it meets $M[13$, p. 134, lemma 8.7]. llence for each $V \in \mathbf{U}$, there exists a $k(U)$ such that the relation $z_{1}^{r}(U) \sim z_{2}^{r}(U)$ on $\bar{U}_{k}$ implies that $z_{1}^{r}(U) \sim z_{2}^{r}(U)$ on $M$. Since $\Phi_{k(v)}^{r} \in I l_{k(v)}^{r}$ implies that $\phi_{k(v)}^{r} \sim 0$ on $\bar{L}_{k(v)}$, it follows that $\phi_{k(v)}^{r} \sim 0$ on $M$. This means that $I_{k(v)}^{r}$ belongs to the kernel of $h_{v}$, and hence $S$ has the property $P_{r}$ with respect to the subset $M$.

9.2. COROLLARY. Under the same hypothesis, hypothesis (i) of Lemma 6.1 is satisfied relative to the subset $M$.

It is now obvious that the result given in 7.4 is applicable here, and hence the set $\gamma^{r}=\left\{\gamma_{i}^{r}\right\}$ is a fundamental system of $r$-cycles as desired.

As a conclusion to this part we give a necessary and sufficient condition that a countable lirh set, $\gamma^{r}=\left\{\gamma_{i}^{r}\right\}$, of $r$-cycles of a compact $G_{\delta}$ subset of a 
locally compact $\mathrm{lc}^{r}$ space $S$ be a fundamental system of $r$-cycles for the subset $M$; that is, $H^{r}(M, \tau)$ is the complete enclosure of the vector space generated by $\Gamma^{r}$.

9.3. THEOREM. Let $M$ be a compact $G_{\delta}$ subset of a locally compact $\mathrm{lc}^{r}$ space S. Suppose further that $\gamma^{r}=\left\{\gamma_{i}^{r}\right\}$ is a countable set of $r$-cycles of $M$ such that for any open set $U$ containing $M$ at most a finite number of elements of $\gamma^{r}$ forms a lirh set on $\bar{U}$ and the remainder are homologous to zero on $\bar{U}$. Then a necessary and sufficient condition that $\gamma^{r}$ be a fundamental system of $r$-cycles for $M$ is that $H_{\Gamma}^{r}(M, \mathfrak{\xi})$, the vector space generated by $\Gamma^{r}$, be dense in $H^{r}(M, \mathfrak{F})$.

Proof. Let $\left\{U_{k}\right\}$ be a sequence of open sets such that $U_{k} \supset U_{k+1}$ and $\cap U_{k}=$ $M$; the existence of this sequence follows from the properties of $M$ and $S$. Then by Theorem 8.2 there exists a sequential decomposition $\left(\mathbf{H}^{r}, \Gamma^{r}\right)$ of $H^{r}(M, \mathfrak{F})$ compatible with $\left\{U_{k}\right\}$.

The necessity follows as an immediate consequence of the remark which follows Corollary 9.2.

To prove sufficiency, proceed as follows. Let $Z^{r} \in H^{r}(M, \mathfrak{F})$; then for $1 / k>$ 0 there exists a $W_{k}^{r} \in H^{r}(M, \mathfrak{F})$ such that $\rho\left(Z^{r}, W_{k}^{r}\right) \leq 1 / k$, which in the present instance implies that $z^{r} \sim w_{k}^{r}$ on $\bar{U}_{k}$. This is true for each integer $k$, and hence we obtain a sequence $\left\{\mathbb{W}_{k}^{r}\right\} \subset H^{r}(M, \mathfrak{F})$. By hypothesis, we have

$$
w_{k}^{r}=\sum_{i=1}^{\infty} a_{k}^{i} \gamma_{i}^{r},
$$

where the on the $\sum$ indicates that all but a finite number of the coefficients $a_{k}^{i}$ are zero. Now let $k_{2}>k_{1}$; then

$$
z^{r} \sim w_{k_{1}}^{r} \sim \sum_{i=1}^{i_{k_{1}}} a_{k_{1}}^{i} \gamma_{i}^{r} \text { on } \bar{U}_{k_{1}}
$$

and

$$
z^{r} \sim w_{k_{2}}^{r} \sim \sum_{i=1}^{i_{k_{2}}} a_{k_{2}}^{i} \gamma_{i}^{r} \quad \text { on } \quad \bar{U}_{k_{2}} \text {. }
$$

Therefore $w_{k_{1}}^{r} \sim w_{k_{2}}^{r}$ on $\bar{U}_{k_{1}}$, and since for $i>i_{k}$ we have $\gamma_{i}^{r} \sim 0$ on $\bar{U}_{k}$, we have

$$
\sum_{i=1}^{i_{k_{1}}}\left(a_{k_{1}}^{i}-a_{k_{2}}^{i}\right) \gamma_{i}^{r} \sim 0 \text { on } \bar{U}_{k_{1}} .
$$


But the $y_{l}^{r}$ are lirh on $\bar{U}_{k_{1}}$ for $i \leq i_{k_{1}}$, and hence $a_{k_{1}}^{i}=a_{k_{2}}^{i}$ for $i \leq i_{k_{1}}$. Continuing this argument, recalling the completeness, and applying Lemma 6.1, we have $Z^{r}=\lim _{k \rightarrow \infty} W_{k}^{r}$, and the proof of the theorem is complete.

\section{NONTRIVIAL 0 - CyCLES}

10. Special fundamental system. In this part, we ultimately establish the existence of a fundamental system of nontrivial 0 -cycles [13, p. 142, Def. 11.4] for compact $G_{\delta}$ subsets of a locally compact 0 -lc space. It has been shown $[6, \mathrm{p} .168$; or 13, p. 142, Th. 11.5] that if $x$ and $y$ are distinct points of a compact space, then there exists a nontrivial 0 -cycle carried by them. We now prove the following lemra.

10.1. LFMMA. If $S$ is a compact space, then for any covering $U$ of $S$, and $\left(j-c y c l e z^{0}\right.$, there exists a refinement $V>U$, and a set of nontrivial 0 -cycles carried by pairs of points, $\gamma_{1}^{0}, \gamma_{2}^{0}, \cdots, \gamma_{k}^{0}$, such that

$$
z^{0}(U) \sim \sum_{i=1}^{k} a^{i} \gamma_{i}^{0}(U), a^{i} \in \xi, \quad \text { on } N(U) \text {. }
$$

l'roof. l.et $U$ be an irreducible refinement [8, p. 247] of $U$. Then on $N(U)$, we have

$$
\begin{array}{r}
z^{0}(U)=a^{1}\left(V_{i_{1}}-V_{i_{2}}\right)+a^{2}\left(V_{i_{2}}-V_{i_{3}}\right)+\cdots+a^{k-1}\left(V_{i_{k-1}}-V_{i_{k}}\right) \\
+a^{k}\left(V_{i_{k}}-V_{i_{k+1}}\right) .
\end{array}
$$

For each $V_{i} \in U$ there exists a $p_{i} \in V_{i}$ such that $p_{i} \notin V_{h}$ if $i \neq h$. For each $m$. let $\gamma_{m}^{0}$ ise a nontrivial 0 -cycle carried by $p_{i_{m}} \cup p_{i_{m+1}}$. Obviously $\gamma_{m}^{0}(U)=$ $V_{i_{m}}-V_{i_{m+1}}$, and hence $z^{0}(U)$ is homologous to $\sum_{i=1}^{i_{1}^{+1}} a^{i} \gamma_{i}^{0}(U)$ on $N(U)$.

11. Existence theorem. The proof of the existence theorem now follows.

11.1. Тнњовні. Let $M$ be a compact $G_{\delta}$ subset of a locally compact 0-lc space $S$. Then there exists a fundamental system of nontrivial 0-cycles carried by pairs of points of $M$ satisfying hypothesis 8.4 for $r=0$.

Proof. Let $\left\{\varepsilon_{k}\right\}$ be a sequence of coverings of $S$ composed of connected open sets such that the part of $\varepsilon_{k}$ that meets $M$ is finite and further $\varepsilon_{k+1}>\varepsilon_{k}$. If $n_{k}$ is the number of components [see 8, p. 15] of the subcollection of $\varepsilon_{k}$ that meets $M$, then we have $n_{k} \leq n_{k+1}$. Since $M$ is a $G_{\delta}$ subset of $S$, then the sequence $\left\{\varepsilon_{k}^{r}\right\}$ can be so selected that, with $E_{k}^{M}$ denoting an element $E_{k} \in \varepsilon_{k}$ 
such that $E_{k} \cap M \neq 0$, we have $\cup E_{k}^{M} \supset \cup E_{k+1}^{M}$ and $\cap\left(\cup E_{k}^{M}\right)=M$.

Let $\left\{p_{j}\right\}\left(j=1, \cdots, n_{1}\right)$ be points of $M$ such that for $j_{1} \neq j_{2}, p_{j_{1}}$ and $p_{j_{2}}$ belong to distinct components of $\varepsilon_{1} \cap M$. Then there exist $n_{1}-1$ nontrivial 0cycles of $M$ with coordinates on the coverings $\varepsilon_{1} \cap M$ forming a lirh set on $N\left(\varepsilon_{1} \cap M\right)$ and consequently on any $\varepsilon \cap M$, where $\varepsilon>\varepsilon_{1}$; hence the cycles themselves are lirh on $M$.

For $\varepsilon_{2} \cap M$, when $n_{2}>n_{1}$, we may add $n_{2}-n_{1}$ nontrivial 0 -cycles to this collection in such a way that they belong to the kernel of $h_{e_{1}}$, but have coordinates on $\varepsilon_{2} \cap M$ which together with the previous set form a lirh set.

A continuation of this process yields a countable lirh set of nontrivial 0 cycles, $\gamma^{0}=\left\{\gamma_{i}^{0}\right\}$, which together with a sequence $\mathbf{H}^{0}$ of subsets of $H^{0}(M, \xi)$ gives rise to a sequential decomposition $\left(\mathbf{H}^{0}, \Gamma^{0}\right)$ compatible with both $\left\{\varepsilon_{k}\right\}$ and $\left\{U_{k}\right\}$, where $U_{k}=U E_{k}^{M}$. This latter follows since $\gamma_{1}^{0}, \cdots, \gamma_{i_{k}}^{0}$ is a basis for cycles on $M$ with respect to homology on $\bar{U}_{k}$, for the covering $\varepsilon_{k} \cap M$ is composed of connected open sets, and coordinates of $\gamma_{i}^{0}\left(i=1, \cdots, i_{k}\right)$ form a maximum lirh set on $\varepsilon_{k} \cap M$. Thus by Theorem 9.3 it remains only to show that the vector subspace $H_{\Gamma}^{0}(M, \tau)$, generated by $\Gamma^{0}$, is dense in $H^{0}(M, \tau)$.

If this is not true, then there exists $Z^{0} \in H^{0}(M, \tilde{F})$ such that $\rho\left(Z^{0}\right.$, $\left.H_{\Gamma}^{0}(M, \xi)\right)>0$. As in Theorem 9.3, for some $k>0$ the cycles $z^{0}, \gamma_{1}^{0}, \ldots$, $\gamma_{i_{k}}^{0}$ are lirh on $\bar{U}_{k}$. Wherefore $[13, \mathrm{p} .167$, Th. 19.2] there exists a covering $U$ of the space such that $z^{0}(U), \gamma_{1}^{0}(U), \cdots, \gamma_{i_{k}}^{0}(U)$ are lirh on $\bar{U}_{k}$ for all $U>U$. By Lemma 10.1 we have the existence of a refinement $U>U$ such that

$$
z^{0}(U) \sim \sum_{j=1}^{s} a^{j} w_{j}^{0}(U), \quad\left(a^{j} \in \tilde{\xi}, s \text { finite }\right)
$$

where the $w_{j}^{0}(U)(j=1, \cdots, s)$ are coordinates on $N(U)$ of nontrivial 0 -cycles $w_{j}^{0}$ of $M$ carried by pairs of points. Since the $w_{j}^{0}$ are homologous to a linear combination of the $\gamma_{i}^{0}$ on $\bar{U}_{k}\left(i=1, \ldots, i_{k}\right)$, we may say the same about the coordinates on $N(U)$, namely

$$
w_{j}^{0}(U) \sim \sum_{i=1}^{i_{k}} \mathfrak{b}_{j}^{i} \gamma_{i}^{0}\left(U^{y}\right)
$$

Thus we have the homology on $\bar{U}_{k}$,

$$
z^{0}(U) \sim \sum_{j=1}^{s} a^{j} \sum_{i=1}^{i_{k}} b_{j}^{i} \gamma_{i}^{0}(U)
$$

in contradiction to what was noted above. Ilence $H_{\Gamma}^{0}(M, \tau)$, as required. 


\section{APPLICATIONS}

12. Related results. As announced in the beginning, the origin of the preceding results lies in an attempt to investigate the problem of imbedding compact spaces in locally connected spaces. Problems of a related nature have already received the attention of various authors. References to these may be found in Wilder [11]; in the latter paper necessary and sufficient conditions are given under which a compact subset of a metric space may be made connected and locally connected by the addition of a set of nonintersecting open arcs with diameters which form a null sequence. Another result in this direction was that of MacKay [9], according to which a Peano continuum may be made 1-connected and locally 1 -connected in the sense of homology by the addition of a denumerable set of nonintersecting open 2-cells with diameters forming a null sequence.

13. Preliminary remarks. By application of the formal results obtained in the earlier parts of this paper, we are able to obtain a partial solution of the imbedding problem which gave rise to this investigation. Before proceeding to the first theorem we set down the following notation.

13.1. NOTATION. If $\gamma^{0}$ is a set of nontrivial 0 -cycles carried by pairs of points of a space $S$, and $V$ is an open set contained in $S$, then by $y^{0} \cap \bar{V}$ is meant the collection of 0 -cycles of $\gamma^{0}$ whose entire carriers are subsets of $\bar{V}$.

14. A sufficient condition. A sufficient condition for the imbeddability of a compact Hausdorff space in a $0-1$ c continuum is given by the following theorem.

14.1. THE OREM. Let $M$ be a compact Hausdorff space and let $\gamma^{0}=\left\{\gamma_{i}^{0}\right\}$ be a countable sequence of nontrivial 0 -cycles carried by pairs of points of $M$, respectively $p_{i_{1}} \cup p_{i_{2}}$, such that: (i) $H^{0}(M, \xi)$ is a Hausdorff space in a topology induced by a sequential decomposition $\left(\mathbf{H}^{0}, \Gamma^{\prime 0}\right)$, where $\gamma^{\prime 0}$ is a subset of $\gamma^{0}$; (ii) if $\mathcal{E}$ is any finite covering of $M$, then diam $\left(p_{i_{1}} \cup p_{i_{2}}\right)<\mathcal{E}[13$, p. 106, Def. 3.5] except for at most a finite number of $i$; (iii) if $U$ is any open set of $M$ and $p \in U$, then there exists an open set $V, p \in V \subset U$, such that $H^{0}(\bar{V}, \tau)$ satisfies hypothesis (i) in terms of a subset of $\gamma^{0} \cap \bar{V}$ (in place of $\left.\gamma^{0}\right)$. Then $M$ is imbeddable as a $G_{\delta}$ subset of a 0 -lc continuum formed by the union of $M$ and a countable set of arcs.

Proof. Let $\left\{a_{i}\right\}$ be a countable collection of disjoint open arcs adjoined to $M$ such that the end-points of $\bar{a}_{i}$ are $p_{i_{1}}$ and $p_{i_{2}}$. L Let $M^{\prime}$ be the set composed of $M \cup A$, where $A=U a_{i}$. We shall topologize $M^{\prime}$ as follows: if $U$ is to be an 
open set of $M^{\prime}$, then $U=U^{*} \cup V^{*}$, where $U^{*}$ is an open subset of $M$ by its original topology, and $V^{*}$ is an open set of $A$, where $A$ is topologized by the convention that every open set of an $a_{i}$ is open in $A$. The set $V^{*}$ may be empty if and only if $U^{*} \subset M-\bar{P}$, where $P$ is the set composed of all carriers $p_{i_{1}} \cup p_{i_{2}}$; $U^{*}$ may be empty under any hypothesis. If $U^{*} \cap P \neq 0$, then $V^{*}$ must contain all but at most a finite number of entire arcs $a_{i}$ such that $p_{i_{1}} \cup p_{i 2} \subset U^{*}$, and complements of closed subsets of the remaining finite number, and it may also contain other sets open in $A$. For each $a_{i}$ such that only one end-point, say $p_{i 1}$, is in $U^{*}, V^{*}$ must contain an open subset of $\bar{a}_{i}$ containing $p_{i_{1}}$.

(a) $M^{\prime}$ is a compact Hausdorff space containing $M$ as a compact $G_{\delta}$ subset. In order to show that $M^{\prime}$ is Hausdorff, it seems evident that we need only verify the separation axiom, and that only for points of $\bar{P}$; the other possibilities have obvious verifications. Therefore let $q_{1}$ and $q_{2}$ be two distinct points of $\bar{P}$. Since $M$ is compact Ilausdorff and hence normal, there exist sets $U_{1}$ and $U_{2}$ such that $\bar{U}_{1} \cap \bar{U}_{2}=0$ and $q_{1} \in L_{1}^{\prime}$ and $q_{2} \in U_{2}$. These sets will correspond to the $U^{*}$ 's of the open sets of $1^{\prime}$. The only difficulty in selecting suitable $V^{*}$ 's comes when $U_{1}$ and $U_{2}$ are such that, for a carrier $\left(p_{i_{1}} \cup p_{i_{2}}\right)$ of some cycle, we have $p_{i 1} \in U_{1}$ and $p_{i 2} \in U_{2}$. Since $M$ is normal, there exist open sets $U_{1}^{\prime}$ and $U_{2}^{\prime}$ such that $U_{1}^{\prime} \cap U_{2}^{\prime}=0, \bar{U}_{1} \subset U_{1}^{\prime}, \bar{U}_{2} \subset U_{2}^{\prime}$. Let $U_{3}^{\prime}=M-\left(\bar{U}_{1} \cup \bar{U}_{2}\right)$; then $U^{\prime}=\left(U_{1}^{\prime}, U_{2}^{\prime}, U_{3}^{\prime}\right)$ is a covering of $M$, and hence by hypothesis we have diam $\left(p_{i_{1}} \cup p_{i_{2}}\right)<U^{\prime}$ except for at most a finite number of carriers, $\left(p_{i_{1}} \cup p_{i_{2}}\right)$, such that $p_{i_{1}} \in U_{1}$ and $p_{i 2} \in U_{2}$. For such a carrier $\bar{a}_{i}^{*}$ can be a closed interval of $a_{i}$ separating $p_{i_{1}}$ and $p_{i_{2}}$ on $\bar{a}_{i}$; and then $\bar{a}_{i}-\bar{a}_{i}^{*}=\alpha_{i 1} \cup \alpha_{i 2}$, two disjoint open sets such that $p_{i_{1}} \in \alpha_{i_{1}}$ and $p_{i_{2}} \in \alpha_{i 2}$. It should now be evident, without further elaboration, that the disjoint open sets $V_{1}^{*}$ and $V_{2}^{*}$, the sets corresponding to the $V^{*}$ of the defining topology, may be chosen so that disjoint open sets of $M^{\prime}$ can be constructed which contain $q_{1}$ and $q_{2}$ respectively; and so $M^{\prime}$ is a Hausdorff space.

The identity mapping is here also a continuous mapping, and hence $M$ as a subset of $M^{\prime}$ is compact. Let $\mathcal{E}$ be any covering of $M^{\prime}$; then a finite subcollection of $\varepsilon$ covers $M$. By the topology of the space $M^{\prime}$, at most a finite number of arcs $a_{i}$ are not completely contained in this subcollection of $\mathcal{E}$. The closures of these arcs are also compact subsets of $M^{\prime}$, and hence evidently there exists a finite subcollection of $\mathcal{E}$ which covers $M^{\prime}$, so that $M^{\prime}$ is a compact Hausdorff space.

Evidently, if $\gamma^{00}$ is the countable subset of hypothesis (i), the $\mathbf{H}^{0}$ of the sequential decomposition can be chosen so that if $H_{k}^{0}+G_{k}^{0}=H^{r}(M, \mathcal{F})$, direct, then $\Gamma_{i}^{\prime 0}(i=1,2, \cdots, k)$ is a base for $G_{k}^{0}$. This selection merely simplifies the construction of the next paragraph. 
We proceed now to the construction of a collection of open sets $\left\{Q_{k}\right\}$ with which $\left(\mathbf{H}^{0}, \Gamma^{\prime 0}\right)$ will be compatible; incidentally, it also shows that $M$ is a $G_{\delta}$ subset of $M^{\prime}$. Let $f_{m}$ be a fixed homeomorphism of the open interval $0<x<1$ onto the open arc $a_{m}$. Then if $\left[a_{m}: 2^{t}\right]$ denotes the complement in $a_{m}$ of the set $f_{m}\left(U x \mid 1 / 2^{t+2}<x<1-1 / 2^{t+1}\right)$, we shall denote by $A_{k}$ the set defined by

$$
\bigcup_{m>k}^{\infty}\left(a_{m} \cup\left[a_{k}: 2^{k}\right] \cup \cdots \cup\left[a_{1}: 2^{k}\right]\right)
$$

This will be the complement in $A$ of a subset closed in $M^{\prime}$ and contained in $A$, so that we may in this way construct a countable collection $\left\{Q_{k}=M \cup A_{k}\right\}$ of open sets closing down on $M$ with the property that $Q_{k} \supset Q_{k+1}$. By the topology of the space $M^{\prime}$ and the construction of $\left\{Q_{k}\right\}$, we may readily see that, with respect to this collection of sets closing down on $M$, the sequence $\gamma^{\prime 0}$ of 0 cycles on $M$ is such that $\gamma_{i}^{\circ 0} \sim 0$ on $\bar{Q}_{k}$ for $i>k$. Furthermore, from the properties of the sequential decomposition $\left(\mathbf{H}^{0}, \Gamma^{\prime 0}\right)$, it is evident that either $\left\{\gamma_{i}^{\prime}{ }^{0}\right\}$ ( $1 \leq i \leq k)$, or a subsequence of it, is a base for 0 -cycles of $M$ relative to homology on $\bar{Q}_{k}$. Hence the desired compatibility is established, and in subsequent parts of the proof it will be possible to apply Theorem 9.1.

(b) $M^{\prime}$ is connected. Now let $z^{0}$ be any 0 -cycle of $M^{\prime}$. By Lemma 10.1, for any covering $U$ there exists a refinement $U>U$ such that

$$
z^{0}(U) \sim \sum_{j=1}^{s} a^{j} g_{j}^{0}(U),
$$

where the $g_{j}^{0}(U)$ are coordinates on the covering $U$ of nontrivial 0 -cycles carried by pairs of points. We must show that $z^{0}(U) \sim 0$. This will amount to showing that each $g_{j}^{0}(U) \sim 0$. Consider now the nontrivial 0 -cycle $g_{j}^{0}$ carried by the pair of points $q_{j 1} \cup q_{j 2}$; there are three possibilities that must be taken into account.

Case 1: $q_{j 1}, q_{j 2} \in M$. Under this hypothesis, by Theorem 9.1 and 7.5 (1) there exists a unique homology, $g_{j}^{0} \sim \sum_{i=1}^{\infty} a^{i} \gamma_{i}^{\prime 0}$. Since $\gamma_{i}^{\prime 0} \sim 0$ on $M^{\prime}$ for all $i$, then, as a consequence of Corollary 9.2, the complete hypothesis of Lemma 6.1 is satisfied, so that $g_{j}^{0} \sim 0$ on $M^{\prime}$ as required for the connectedness of $M^{\prime}$.

Case 2: $q_{j 1}, q_{j 2} \in A$. Suppose $q_{j 1} \in a_{m}$ and $q_{j 2} \in a_{n}$. Then evidently $g_{j}^{0} \sim z_{m n}^{0}$, where $z_{m n}^{0}$ is a nontrivial 0 -cycle carried by a pair of points, $p_{m 1} \cup$ $p_{n 1}$ (there are points of the carrier sets of 0 -cycles in $\gamma^{0}$ ). Vence $z_{m i n}^{0}$ is a 0 -cycle of $M$, and we may proceed as above with the sane result. 
Case 3: $q_{j 1} \in M$ and $q_{j 2} \in A$. If $q_{j 2} \in a_{i}$, then $g_{j}^{0} \sim z_{1 i}^{0}-z_{21}^{0}$, where $z_{1 i}^{0}$ is a nontrivial 0 -cycle carried by the pair $q_{j_{1}} \cup p_{i_{1}}$, and $z_{21}^{0}$ is a nontrivial 0 -cycle carried by the pair $p_{i_{1}} \cup q_{j_{2}}$. Evidently $z_{21}^{0} \sim 0$ on $M^{\prime}$; hence $g_{j}^{0} \sim z_{1 i}^{0}$ on $M^{\prime}$, where $z_{1 i}^{0}$ is on $M$. Again we may proceed as above with the same result.

Thus we have shown that in all possibilities we have $g_{j}^{0}(U) \sim 0$ on $M^{\prime}$, so that the space $M^{\prime}$ is connected.

(c) The space $M^{\prime}$ is 0 -lc. To examine $M^{\prime}$ for 0 -local connectedness, it is evident that we need only investigate neighborhoods of $M$. Let $p \in M \subset M^{\prime}$; and let $U$ be any open subset of $M^{\prime}$ containing $p$. Then by the topology of $M^{\prime}$, we have $U=U^{*} U V^{*}$, where $p \in U^{*}$; further, by the hypothesis, there exists a $U_{1}^{*} \subset U^{*}$ such that $H^{0}\left(\bar{U}_{1}^{*}, \mathcal{F}\right)$ is a Hausdorff space under a topology induced by a sequential decomposition based on a subset of $\gamma^{0} \cap \bar{U}_{1}^{*}$. Let $V_{1}^{*}$ be a set contained in $V^{*}$ such that $\bar{V}_{1}^{*} \subset U$, and containing all but a finite number of entire arcs $a_{i}$ corresponding to carriers of the subset of $\gamma^{0} \cap \bar{U}_{1}^{*}$ just above, and complements of $a_{i}$ of closed intervals of the remaining finite number. Then $U_{1}=$ $U_{1}^{*} \cup V_{1}^{*} \subset U$. Let $k$ be the finite number of arcs $a_{i}$ such that the entire arc fails to belong to $U$. Then for any covering $U$ (by the same process as was used above in proving the connectedness of $\left.M^{\prime}\right)$, at most $2 k 0$-cycles of $U_{1}$ are lirh in $U$; hence by 8.1 the space $M^{\prime}$ is $0-1$.

15. The class $\mathcal{E}$. In order to improve the value of the preceding theorem, it is desirable that the class $\Re$ of compact spaces which satisfy the hypothesis includes, in addition to all metric spaces, a definite nonmetric space. This is partially accomplished by showing that the nonmetric compact Hausdorff space of Alexandroff-Urysohn [used in another problem, 1, p. 76] fulfills the requirements of the theorem; details of this procedure are tedious, though not difficult, and will not be given here. Using practically the same technique, we can show that the class of all compacta is a subset of $\AA_{\text {. }}$ Of course this last result yields immediately the conclusion that every compactum is imbeddable as a compact $G_{\delta}$ subset of a 0 -lc continuum formed by the addition of a countable set of arcs; this is also a corollary of the Urysohn theorem on the imbedding of such spaces in the Hilbert parallelotope.

16. A necessary condition. In giving a necessary condition, we shall restrict ourselves to separable ${ }^{1}$ compact $G_{\delta}$ subsets. Incidentally, we might remark that the Alexandroff-Urysohn nonmetric space, referred to above, is separable; this indicates that the restriction imposed does not limit the spaces

\footnotetext{
${ }^{1}$ Because of the occasional variety that is observed in the use of this term, we note that in this paper a separable space will be one having a countable dense subset.
} 
to which the theorem is applicable too sharply. As an immediate consequence of an obvious lemma and a theorem due to Wilder [13, p. 143, Th. 11.10], we may state the following theorem.

16.1. THEOREM. If $S$ is a compact 0 -lc space, $U$ is an open set of $S$, and $p, q \in U$, such that a nontrivial cycle carried by $p \cup q$ bounds in $U$, then $p \cup q$ lies in a connected 0-lc subset of $U$.

The necessary condition is given in the following theorem.

16.2. THEOREM. Let $M$ be a separable compact $G_{\delta}$ subset of a locally compact space $S$. If $M$ is a subset of a 0 -lc open subset of $S$, then there exists a countable $\gamma^{0}=\left\{\gamma_{i}^{0}\right\}$ of nontrivial 0 -cycles of $M$ carried by pairs of points such that: (i) if $p \in M$ and $U$ is any open set containing $p$, then there exists a $V \mathbb{C} U, p \in V$, such that a subset $\gamma_{v}^{0}$ of $\gamma^{0} \cap(\bar{V} \cap M)$ gives rise to a sequential decomposition $\left(\mathbf{H}^{0}, \Gamma_{v}^{0}\right)$ which induces a Hausdorff topology in $H^{0}(\bar{V} \cap$ H, F); (ii) all but a finite number of carriers of the 0-cycles of $\gamma_{v}^{0}$, for any open set $P \supset \bar{V} \cap M$, are contained in 0-lc connected open sets of $P$.

Proof. Let $M^{*}$ be a countable dense subset of $M$, and let $\gamma^{0}$ be the collection of all nontrivial 0 -cycles carried by pairs of points of $M^{*}$. Let $p$ be an arbitrary point of $M$, and $U$ any open set of $S$ containing $p$. Then $\bar{U}^{*}=\bar{U} \cap M$ is a compact subset of $M$. We may then select a set $V$, open in $M$, containing $p$ and such that $\bar{V} \subset U \cap M$.

Consider now a countable family of coverings $\left\{\varepsilon_{i}\right\}$, relative to the 0 -lc open subset of $S$ containing $M$, each consisting of connected open sets and such that the portion on $\bar{V}$ of any $\varepsilon_{i}$ is the star-closure refinement ${ }^{2}$ of all its predecessors, and further such that if $c_{i}$ is the number of components of the covering $\varepsilon_{i} \cap \bar{V}=$ $\varepsilon_{i}^{*}$, then $c_{i}>c_{j}$ for $i>j$. We shall now select a sequence of points of $\bar{V} \cap M^{*}$ as follows: let the components of $\varepsilon_{i}^{*}$ be arbitrarily ordered, $C_{1}, C_{2}, \ldots, C_{c_{1}}$; then the components of $\varepsilon_{2}^{*}$ are indexed $C_{c_{1}+1}, \ldots, C_{c_{1}}+c_{2}$, in such a way that those which are contained in $C_{1}$ are indexed first; then the indexing proceeds to those of the remainder contained in $C_{2}$, and so on. A continuation of this process gives a countable sequence of elements, $\mathbf{C}=\left\{C_{j}\right\}$. Now in each element $C_{j}$ choose a point, $p_{j} \in \bar{V} \cap M^{*}$, in such a way that (1) at most one $p_{j} \in C_{k}$ $(j \leq k)$, and (2) when a previously selected $p_{j} \in C_{k}(j<k)$, then the selection of a new $p_{k}$ is omitted. With pairs of $p_{j}^{\prime s}$ as carriers, we may associate nontrivial 0 -cycles $\gamma_{j}^{0}$, carried by the pair $p_{j} \cup p_{k}$, where the pairing is made as follows: if $\varepsilon_{i}^{*}$ is the first covering of the sequence of coverings $\left\{\varepsilon_{i}^{*}\right\}$, such that $p_{j}$ and

${ }^{2}$ If $\operatorname{St}(U, U)$ is a closure refinement [13, p. 133, Def. 8.5] of a covering $U$, then $U$ is called a star-closure refinement of $\dot{U}$. 
$p_{k}$ belong to distinct components of $\varepsilon_{i}^{*}$, then $p_{j}$ is paired to $p_{k}$ if and only if they belong to the same component of $\varepsilon_{i-1}^{*}$; this will give us a countable ordered subcollection $\gamma_{v}^{0}$ of $\gamma^{0} \cap \bar{V}$, the elements of which have the following properties: (1) the set $\gamma_{v}^{0}$ is lirh on $\bar{V}$, (2) for any covering $\varepsilon_{i}^{*}$, the coordinates of the first $c_{i}-1$ of the 0 -cycles are lirh on the covering $\varepsilon_{i}^{*}$ and form a basis for homology on the covering, and the remainder are homologous to zero. Consequently we may show in a way similar to that used in the proof of Theorem 11.1 that this collection gives rise to a sequential decomposition which induces a Hausdorff topology in $H^{0}(\bar{V}, \tau)$.

The remainder of the proof follows easily. By a theorem due to Begle [2, p. 63] all but a finite number of 0 -cycles of the collection $\gamma_{v}^{0}$ bound in $P$, so that by Theorem 16.1 all but a finite number of the carriers of the 0 -cycles of this collection are contained in 0 -lc connected sets of $P$.

Since the $0-1 \mathrm{c}$ property is a local characteristic of a space, we could give an obvious localization of the preceding result with the natural satisfaction of aesthetic completeness.

\section{REFERENCES}

1. P. S. Alexandroff and P. Urysohn, Mémoire sur les espaces topologiques compacts, Verh. Nederl. Akad. Wetensch. Verslagen, Afd. Natuurk. Sect. 1, 14 (1929), 1-96.

2. E. G. Begle, Locally connected spaces and generalized manifolds, Amer. J. Math. 64 (1942), $553-574$.

3. $59-70$.

Duality theorems for generalized manifolds, Amer. J. Math. 67(1945),

4. N. Bourbaki, Algèbre, Paris, 1942-.

5. , Topologie générale, Paris, 1940-.

6. E. Čech, Théorie générale de l'homologie dans un espace quelconque, Fund. Math. 19 (1932), $149-183$.

7. F. Hausdorff, Mengenlehre, Berlin, 1927, 1935.

8. S. Lefschetz, Algebraic topology (Amer. Math. Soc. Colloq. Pub. 27), New York, 1942.

9. R. MacKay, Planarity of Peano spaces in terms of homology bases, unpublished Michigan dissertation, 1938.

10. G. T. Whyburn, Analytic topology (Amer. Math. Soc. Colloq. Pub. 28), New York, 1942.

11. R. L. Wilder, On the imbedding of subsets of a metric space in Jordan continua, Fund. Math. 19 (1932), $45-64$.

12. $52(1946), 446$.

, Homology groups of perfectly normal spaces, Bull. Amer. Math. Soc.

13. 1949.

Topology of Manifolds (Amer. Math. Soc. Colloq. Pub. 32), New York, 


\section{PACIFIC JOURNAL OF MATHEMATICS}

\section{EDITORS}

R. M. RoBINSON

University of California Berkeley 4, California

\section{*R. P. Dilworth}

Califomia Institute of Technology Pasadena 4, California

\section{E. F. BECKENBACH, Managing Editor}

University of California

Los Angeles 24, California

*During the absence of Herbert Busemann in 1952.

\section{ASSOCIATE EDITORS}

\author{
R. P. DILWORTH \\ HERBERT FEDERER \\ MARSHALL HALL
}

\author{
P. R. HALMOS \\ HEINZ HOPF
}

R. D. JAMES

\author{
BØRGE JESSEN \\ PAUL LÉVY \\ GEORGE PÓLYA
}

\author{
J. J. STOKER \\ E. G. STRAUS
}

KÖSAKU YOSIDA

\section{SPONSORS}

UNIVERSITY OF BRITISH COLUMBIA

CALIFORNIA INSTITUTE OF TECHNOLOGY

UNIVERSITY OF CALIFORNIA, BERKELEY

UNIVERSITY OF CALIFORNIA, DAVIS

UNIVERSITY OF CALIFORNIA, LOS ANGELES

UNIVERSITY OF CALIFORNIA, SANTA BARBARA

OREGON STATE COLLEGE

UNIVERSITY OF OREGON

\author{
UNIVERSITY OF SOUTHERN CALIFORNIA \\ STANFORD UNIVERSITY \\ WASHINGTON STATE COLLEGE \\ UNIVERSITY OF WASHINGTON \\ AMERICAN MATHEMATICAL SOCIETY \\ NATIONAL BUREAU OF STANDARDS, \\ INSTITUTE FOR NUMERICAL ANALYSIS
}

Mathematical papers intended for publication in the Pacific Journal of Miathematics should be typewritten (double spaced), and the author should keep a complete copy. Manuscripts may be sent to any of the editors. All other communications to the editors should be addressed to the managing editor, E. F. Beckenbach, at the address given above.

Authors are entitled to receive 100 free reprints of their published papers and may obtain additional copies at cost.

The Pacific Journal of Mathematics is published quarterly, in March, June, September, and December, by the University of California, Berkeley 4, California. The price per volume (4 numbers) is $\$ 8.00$; single issues, $\$ 2.50$. Special price to individual faculty members of supporting institutions and to individual members of the American Mathematical Society: $\$ 4.00$ per volume; single issues, $\$ 1.25$.

Subscriptions, orders for back numbers, and changes of address should be sent to the publishers, University of California Press, Berkeley 4, California.

Printed at Ann Arbor, Michigan. Entered as second class matter at the Post Office, Berkeley, California.

\section{UNIVERSITY OF CALIFORNIA PRESS • BERKELEY AND LOS ANGELES}




\section{Pacific Journal of Mathematics}

\section{Vol. 2, No. $2 \quad$ February, 1952}

L. Carlitz, Some theorems on Bernoulli numbers of higher order...

Watson Bryan Fulks, On the boundary values of solutions of the heat equation........................................ 141

John W. Green, On the level surfaces of potentials of masses with fixed center of gravity................................... 147

Isidore Heller, Contributions to the theory of divergent series .......... 153

Melvin Henriksen, On the ideal structure of the ring of entire functions . . . 179

James Richard Jackson, Some theorems concerning absolute neighborhood retracts........................................ 185

Everett H. Larguier, Homology bases with applications to local connectedness ................................... 191

Janet McDonald, Davis's canonical pencils of lines ................ 209

J. D. Niblett, Some hypergeometric identities . . . . . . . . . . . . . . . . . . 219

Elmer Edwin Osborne, On matrices having the same characteristic equation...................................... 227

Robert Steinberg and Raymond Moos Redheffer, Analytic proof of the

Lindemann theorem ................................. 231

Edward Silverman, Set functions associated with Lebesgue area ......... 243

James G. Wendel, Left centralizers and isomorphisms of group algebras . . 251

Kosaku Yosida, On Brownian motion in a homogeneous Riemannian space. 